\title{
Architecture of an Integrated AMR Current Sensor (IACS) System for a Wide Range of Automotive Applications
}

\author{
Schreiber-Prillwitz, Wolfgang*; Nau, Christian** \\ *Elmos Semiconductor AG; **Sensitec $\mathrm{GmbH}$ \\ ${ }^{*}$ Heinrich-Hertz-Str. 1; **Georg-Ohm-Straße 11 \\ *44227 Dortmund; **35633 Lahnau \\ Germany
}

\section{Introduction and General Description}

As an alternative or a replacement for Hall-sensors, AMR-based sensors are increasingly entering the field of angular and linear position measurement, as well as current measurement. For current measurement, Hall-effect based sensors normally are utilizing an iron core to concentrate the magnetic field lines to the sensor area. This flux concentrator typically encloses the primary current line, with the Hall-element placed in the air-gap of the flux concentrator. This construction leads to a perpendicular orientation of the concentrated magnetic field lines to the sensor surface, and by this method to a sufficient signal. Hall-based systems without flux concentrator do need a primary current line inside their housing to enable the now needed small distance between Hall-element and current line to achieve a sufficient signal. The necessary small distance on one hand decreases the dielectric strength of those products, on the other hand the fix cross section of the integrated primary current line determines the maximal allowed current. The bandwidth range of Hall-effect based current sensors is typically 50 to $150 \mathrm{kHz}$. In general, to cover different ranges of current, different products are needed.

The described AMR-based current sensor exhibits no hysteresis as observed in iron core based Hall-sensor solutions. Due to the high sensitivity of AMR sensors, a flux concentrator is not necessary. It is designed for high resolution and very fast electronic measurement from DC up to $500 \mathrm{kHz}$ AC. Contrary to Hall-effect based sensors, the described system enables a differential magnetic field measurement by an advanced geometry of the magnetoresistive elements. Due to this construction the sensor is immune to homogeneous interference fields.

By variation of the geometry of the external primary current bar, this system can be adapted to different current ranges and applications. With this, only one system is needed for a wide range of applications.

The system accuracy can be improved by using either the internal or an external reference voltage. This further reduces temperature drift, and several sensors can share the same reference voltage. The adjustable over-current detection enables fast response in overload situations to prevent damage to e.g. power units.

\section{Demands of Future Applications}

The development e.g. in the field of regenerative energy generation will bring a lot of new application fields for current measurement. Power inverters for photovoltaic panels, or current monitoring in the field of electro mobility (motor control, battery charging and monitoring) are only the most obvious ones. Fact is, that future applications will need to monitor currents in the range from Amperes up to several hundred Amperes, at high bandwidth and simultaneous high dielectric strength. This demands a product with high accuracy and inherent high flexibility.

\section{IACS System Concept}

The new sensor system developed by Sensitec and Elmos, based on the anisotropic magnetoresistive effect (AMReffect), fulfills these demands. It consists of the AMR sensitive sensor cell, the signal conditioning circuitry and two biasing permanent magnets. The latter are for maintaining the initial magnetization direction of the AMR structures

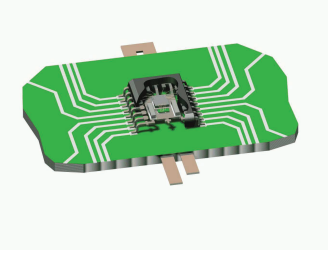

Fig. 1: IACS system concept

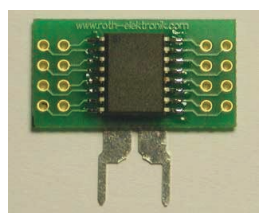

Fig. 2: reflow soldered IACS

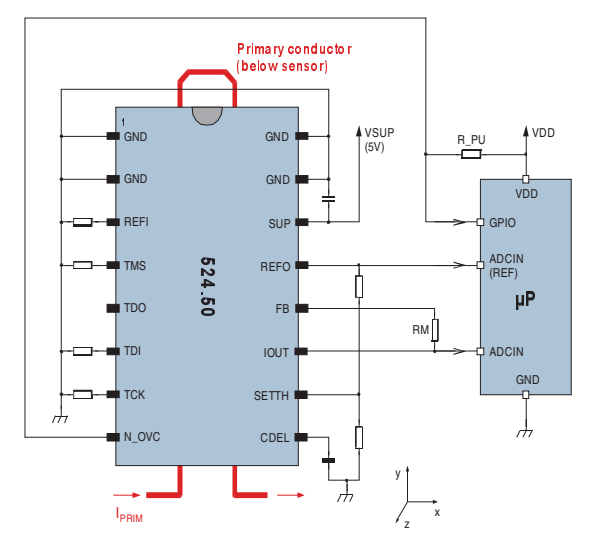

Fig. 3: typical application after the application of very strong over-current spikes. The permanent magnet raw material and the AMRsensitive sensor material are applied on wafer substrates by a special process, thus can be processed further on with standard semiconductor methods, concerning singulation or assembly.

A special leadframe as well as an advanced assembly technique enables a 'system-in-package' solution (SIP): all system components are overmolded within a JEDEC compliant SOIC16 package (Fig. 1). The product can be mounted with standard pick-andplace equipment onto a $\mathrm{PCB}$, and 
subsequently reflow soldered (Fig. 2).

The sensor is measuring the field gradient of a magnetic field $\mathrm{H}$. The field gradient could in general be created by two arbitrarily shaped current lines with opposed current flow direction. This primary current line typically is U-shaped, with its straight parallel parts underneath the sensor (Fig. 1 to 3). Due to the high sensitivity of the AMR sensor a flux concentrator is not needed, with still keeping an excellent signal resolution. The external primary current line concept provides on one side a very good dielectric isolation to the sensor, and secondly gives a degree of freedom concerning the application specific adaptation of the system. For low currents, the primary current line could be placed directly underneath the current sensor package, on the top side of the PCB. For high currents in the range of 200A, thick copper bars are used as conductors onto which the PCB with the current sensor on top shall be glued.

\section{Signal Conditioning Concept}

The magnetic field is changing the resistivity of the elements of the Wheatstone bridge. This imbalance of the bridge results in a differential voltage which will be amplified by the read-out IC and converted to a proportional current at the output of the IC. The IC is feeding this proportional current into the compensation current line of the sensor cell,

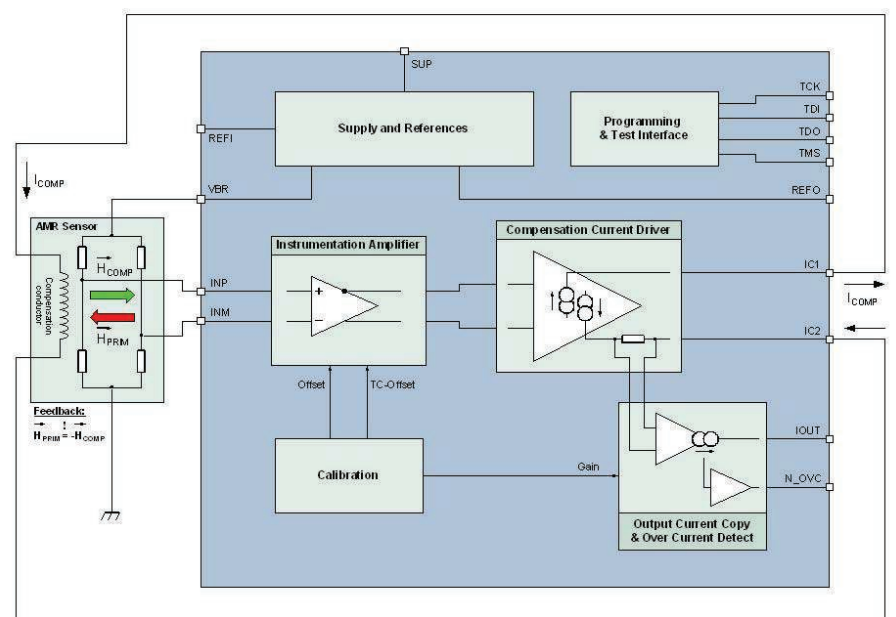

Fig. 4: Block diagram of the signal conditioning circuitry which will create a magnetic field of the same magnitude, but opposite direction as the field generated by the primary current line at the sensor position. A block diagram of the read out IC is given in Fig. 4. With this closed loop principle, high linearity and zero temperature dependency of sensitivity is achieved, as the sensor always is working close to zero excitation. This contributes significantly to the high accuracy of the system. The use of fast, low noise amplifiers allows short step response times $<1 \mu$ s. The output noise of $<1.5 \mu \mathrm{A}$ eff. within a bandwidth of $200 \mathrm{kHz}$ results in an SNR of $>66$ $\mathrm{dB}$. An overload of 3 -times the nominal current is allowed for short pulse times $(<50 \mathrm{~ms})$, in which the system still delivers precise output. Together with the high bandwidth, not only average values, but also fast current transients can be measured. This opens the field for time critical applications like e.g. current sensing in IGBT-bridges used in power inverters.

\section{Technical Base of the IACS}

The AMR effect describes the variation of the specific resistivity of a material, depending on the angle $\xi$ between an

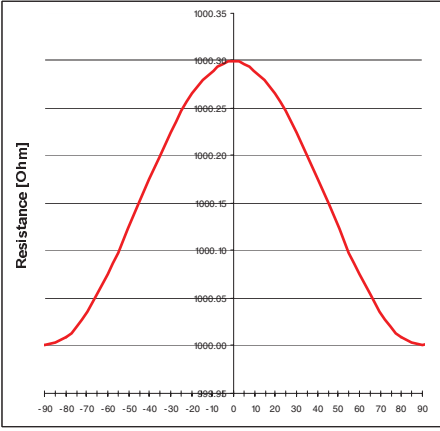

Fig. 5: resistivity (2\%) vs. angle between current and external $\mathrm{H}$-field

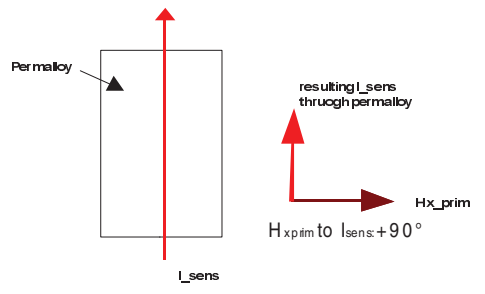

Fig. 6: current and magnetic field w/o barber poles electrical current through this material and an external magnetic field. If the external magnetic field and the current through the material are parallel $\left(0^{\circ}\right)$, the specific resistivity reaches its maximum: $R=R_{\max }$; in case of perpendicular orientation $\left(90^{\circ}\right), R=R_{\min }$. The typical variation of the resistivity is in the range of 1 to $3 \%$. The relation between the effective magnetic field and the current through the AMR-sensitive structure follows the equation:

$$
\rho(\xi)=\rho\left(90^{\circ}\right)+\Delta \rho^{*} \cos ^{2} \xi
$$

In Fig. 5, the red curve represents this formula for a resistivity change of $2 \%$ between parallel and perpendicular current and magnetic field. At $0^{\circ}$, the resistor reaches its maximum value, and at $90^{\circ}$ between $\mathrm{I}$ and $\mathrm{H}$ its minimum. The quadratic characteristic does not allow to recognize the direction of the external $\mathrm{H}$-field. The AMR sensitive structure of the IACS

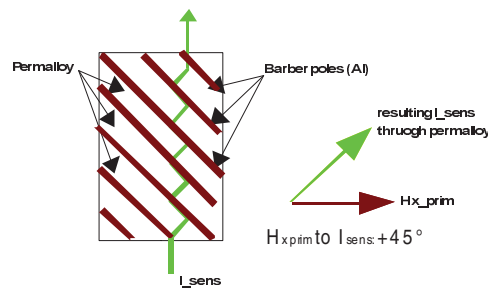

Fig. 7 current and magnetic field with barber poles

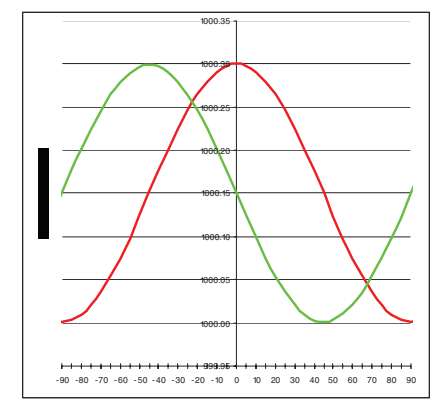

Fig. 8: effect of barber poles 
consists of a permalloy, which has a natural form anisotropy concerning its magnetization due to its dimensions (some $10 \mathrm{~nm}$ thin, some $\mu \mathrm{m}$ wide, several $10 \mu \mathrm{m}$ long). The magnetic domains are oriented along the longitudinal axis of the structure. The effective magnetization results from this natural internal magnetization and the applied external magnetic field. As the internal magnetization has no preferred direction along the longitudinal axis, a flipping of $180^{\circ}$ could occur due to overcurrent spikes. This would lead to a different effective magnetic field, and so to a different sensitivity of the system. In order to always orient the internal magnetization to a defined direction, the biasing magnets are needed. A rotation of the current through the AMR-sensitive resistors of $45^{\circ}$ towards the length axis leads to a more linear transfer function around $0^{\circ}$ (Fig. 8, green curve), and gives additionally information about the direction of the field. This rotation can be achieved by so called 'barber poles'. These are realized as metal lines (Al) under $45^{\circ}$ upon the long axis of the permalloy material. The rather higher conductivity of the Al-lines compared to the permalloy material leads to the effect of a rotation of the current through the permalloy layers (Fig. 6 to Fig. 8).

\section{Principle of Operation}

The current sensor is built as a Wheatstone bridge with a certain geometric set up: both half bridges are located in areas with equal distance from a symmetry axis of the sensor (Fig. 9 and Fig. 9).

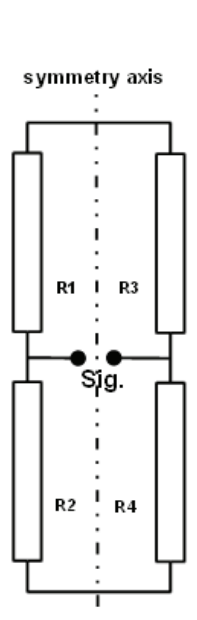

Fig. 9: Wheatstone bridge set up

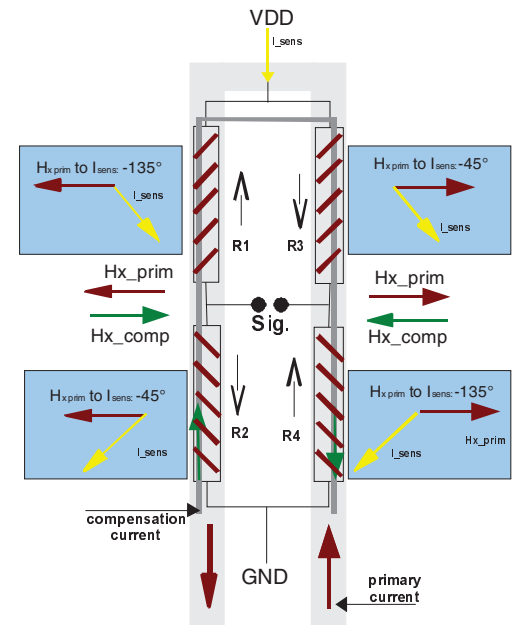

Fig. 10: angular orientation between the current through the resistor and $\mathrm{H}_{\mathrm{x} \_ \text {prim }}$

The gradient of a $\mathrm{H}$-field over this distance within the plane of the sensor gives the output signal. In Fig. 10, the schematic setup is shown. The dark red arrows show the direction of the primary current flow. The primary current bar (light gray) is U-shaped, so that both branches of the bridge do see an opposite oriented $\mathrm{H}$-field $\mathrm{H}_{\text {x_prim. }}$.

The different barber pole orientation within each branch $(\mathrm{R} 1+\mathrm{R} 2, \mathrm{R} 3+\mathrm{R} 4)$ leads to a contrary variation of the resistivity within the branch, and so effectively raises the sensitivity. The trend of the resistivity change is indicated by the black arrows. With resistor values around $1 \mathrm{kOhm}$ and a change of about $2 \%$ the calculation of the Wheatstone bridge output signal gives a full scale signal of approximately $60 \mathrm{mV}$ at supply $\mathrm{UO}=5 \mathrm{~V}$. The position of the symmetry axis of sensor and primary current bar needs to be identically to achieve symmetric conditions within the two branches of the AMR sensor. The effect of an offset between the symmetry axis of sensor and primary current bar is shown as the signal trend along the $\mathrm{x}$-axis in Fig. 13 and Fig. 15.

\section{Gradient Measurement}

The design and the geometric placement of the primary current bar has a big influence on the field gradient over the

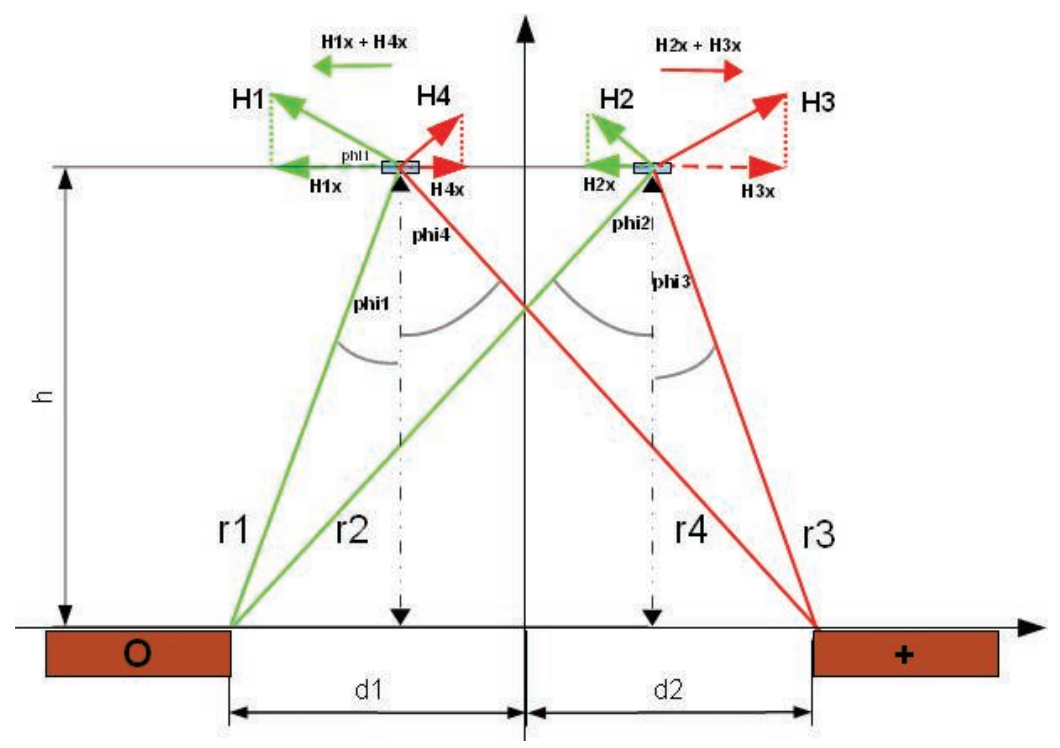

Fig. 11: effective $\mathrm{Hx}$-field at the two half bridges of the sensor sensitive area. The recommended $\mathrm{U}$ shaped conductor contributes with four $\mathrm{H}$-field vectors to the resulting magnetic field at the sensor, as is illustrated in Fig. 11. The light blue upper bars represent each one branch of the bridge (see also Fig. 9 and Fig. 10). The brown bars show the cross section of the primary current bar with a distance $\mathrm{d} 1$ and $\mathrm{d} 2$ to the symmetry axis of the sensor. The orientation of $\mathrm{d} 1$ and $\mathrm{d} 2$ represent the $\mathrm{x}$ - axis in the following diagrams. If $\mathrm{d} 1$ and $\mathrm{d} 2$ are identically, the symmetry axis of sensor and current bar are aligned, and the geometrically offset is zero.

In this simplified sketch it can be seen, that each part of the sensor sees a different resulting $\mathrm{H}_{\mathrm{x}}$-component of the magnetic field generated by the primary current bar. The sum of both absolute values represents the 
magnetic field gradient over the distance of the AMR-sensitive areas of the Wheatstone bridge configuration.

\section{Immunity Against Stray Fields}

One big advantage of measuring the field difference between the two positions of the half bridges is the immunity against external stray fields.

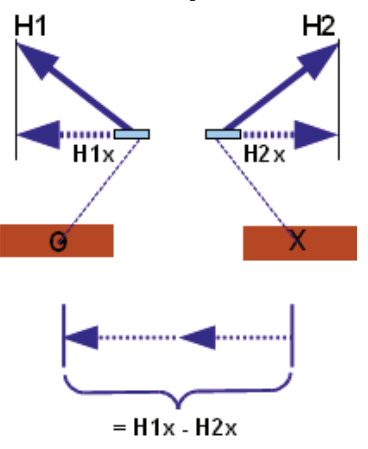

Fig. 12: effect of magnetic stray fields

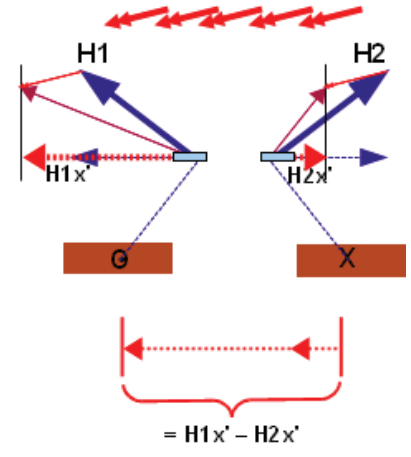

b) resulting disturbed gradient $\mathrm{Hx}$

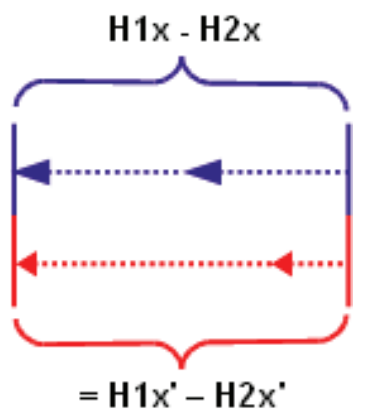

C) comparison of both resulting grad $\mathrm{Hx}$

Fig. 12 a) shows the resulting field gradient $\mathrm{Hx}$ in the undisturbed case. The effect of a stray field is shown in Fig. $12 b$ ). Its components (red arrows) are adding the same magnitude to the Hx vector component for each side of the sensor, and the resulting magnetic field gradient keeps the same ( Fig. $12 \mathrm{c}$ ) under the assumption, that the stray field is homogenous over the area of the sensor chip. This assumption is plausible due to the small distance between the resistors in the $1 \mathrm{~mm}$-range.

\section{Realizing a Wide Range of Applications}

The concept of an external primary current bar gives a certain degree of freedom to adapt the system to a wide range of applications. To get best SNR, the field gradient should be near $1600(\mathrm{~A} / \mathrm{m}) / \mathrm{mm}$ for the full scale range of the

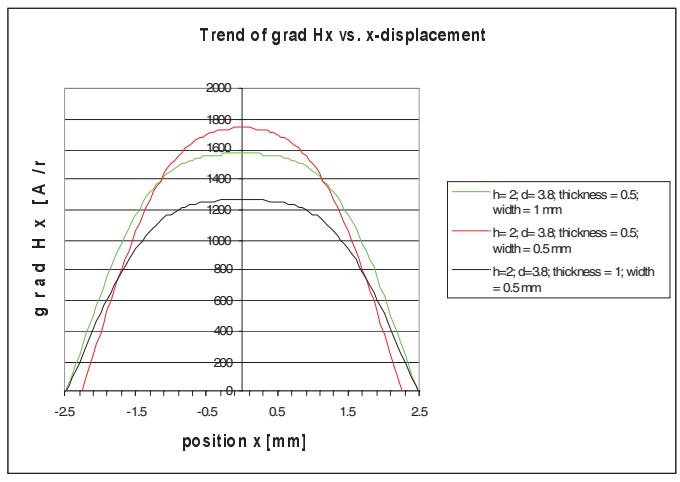

Fig. 13: trend of grad $\mathrm{Hx}$ for $\mathrm{I}_{\text {nom }}=50 \mathrm{~A}$ and different prim. current line cross sections

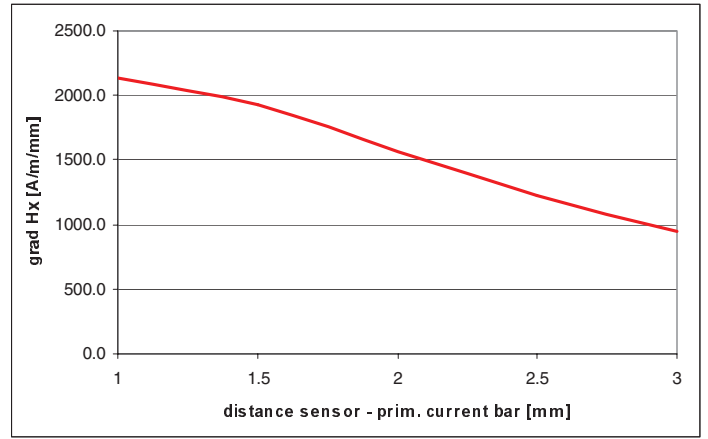

Fig. 14: trend of grad Hx vs. vertical distance for $\mathrm{I}_{\mathrm{nom}}=50 \mathrm{~A}$ primary current in a symmetrical arrangement of primary current bar and sensor area. A second aspect becomes obvious from Fig. 11. The lateral expansion (width and thickness) of the primary current bar can not be neglected, as the different parts of the cross section will contribute to the resulting magnetic field at the sensor. So in reality, it is not sufficient to only calculate the magnetic field after the Biot-Savart formula for a thin linear wire, but to integrate over the cross section of the primary current line. This is even more important for current lines for high currents (several hundred Amperes), whose cross sections will have dimensions in the range of $\mathrm{mm}$, and so are in the same range as the sensor geometry itself. The characteristics of the gradient within the $x-y$-plane should be constant within an area of typical lateral assembly deviations, as this would lead to only small variation in sensitivity. Fig. 13 shows the trend of the magnetic field gradient in $A / \mathrm{m} / \mathrm{mm}$, when the sensor would be moved along the $\mathrm{x}$-axis. This represents the influence of lateral displacement between sensor and primary current bar (with $\mathrm{X}=0$ as the symmetric target configuration in Fig. 9). Additionally, in Fig. 13 the cross section of the primary current bar is changed (see legend). The green line would be the chosen geometry, as it has a low variation to symmetry displacement around $x=0$, and comes closest to the target value of $1600 \mathrm{~A} / \mathrm{m} / \mathrm{mm}$.

A certain variation of the gradient value due to assembly related vertical distance variations can not be avoided. The typical characteristic of grad Hx vs. vertical distance between sensor and current bar (using the primary current line geometry of the green curve in Fig. 13) is shown in Fig. 14. Due to the mechanical construction of the system, the minimum possible distance between primary current line and sensor chip is $1 \mathrm{~mm}$ (from bottom side of the package to surface of the AMR sensor). The decrease of grad $\mathrm{H}_{\mathrm{x}}$ is quite linear for increasing distance. This makes it easy to estimate the sensitivity variation due to 
vertical assembly deviations, and with the possibility to do an end-of-line calibration of the gain by EEPROM programming after assembly, this effect can be compensated.

In Fig. 13 it can bee seen that the thickness of the primary current line is a sensitive parameter concerning the value of the field gradient (red: thickness $=0.5 \mathrm{~mm}$, green: thickness $=0.5 \mathrm{~mm}$, black: thickness $=1 \mathrm{~mm}$ ), in correlation to the grad Hx trend vs. vertical distance shown in Fig. 14. In general, the design of a primary current line has to take into account the current load a certain cross section of a conductor can carry, especially concerning temperature rise. The IPC-2221 for instance gives guidelines how to calculate the cross sections for copper current lines embedded into PCB or attached onto the PCB surface $(I<35 \mathrm{~A}$, trace width $<10 \mathrm{~mm}$; beyond these limits the formular extrapolates the values). For a given current line thickness, temperature rise, and current load, the needed width is calculated after

$$
w[m m]=\frac{I}{\left(k * \Delta T^{b}\right)^{\frac{1}{c}} * t[m m]}
$$

with $\mathrm{t}=$ thickness, and $\mathrm{k}, \mathrm{b}$, and $\mathrm{c}$ constants resulting from fitting to the IPC-2221 curves (source: http://www.circuitcalculator.com/wordpress/2006/01/31/pcb-trace-width-calculator/).

For IPC-2221 internal layers: $k=0.024, b=0.44, c=0.725$,

for IPC-2221 external layers: $k=0.048, b=0.44, c=0.725$.

Table 1 shows the calculated minimum trace width after this formular for a nominal current of $25 \mathrm{~A}$ for an external copper current line. Fig. Fig. 15 gives the according grad $\mathrm{Hx}$ trend for this geometry.

\begin{tabular}{|l|l||l|}
\hline current & 25 & A \\
\hline Cu-thickness & 0.4 & $\mathrm{~mm}$ \\
\hline \hline T- increase & 20 & ${ }^{\circ} \mathrm{C}$ \\
\hline \hline min. trace width & 1.5 & $\mathrm{~mm}$ \\
\hline \hline vertical distance & 1.5 & $\mathrm{~mm}$ \\
\hline distance between arms & 1.2 & $\mathrm{~mm}$ \\
\hline \hline max. grad $\mathrm{H}_{\mathbf{x}}$ & 1500 & A/m/mm \\
\hline \hline
\end{tabular}

Table 1: Dimensions and geometry for Inom $=25 \mathrm{~A}$

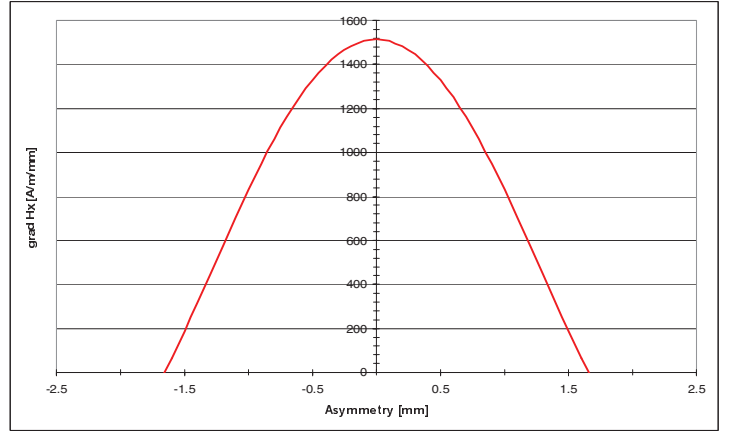

Fig. 15: trend of grad $\mathrm{Hx}$ for $\mathrm{I}_{\text {nom }}=25 \mathrm{~A}$

From this pure theoretical point of view it is possible to realize a current measurement system for $I_{\text {nom }}$ ranges from $0 A$ to $25 \mathrm{~A}$ up to ranges above $0 \mathrm{~A}$ to $200 \mathrm{~A}$, by having the full signal resolution.

\section{Target Performance}

\begin{tabular}{|l||l|}
\hline \multicolumn{1}{|c||}{ Parameter } & \multicolumn{1}{c|}{ IACS 524.50 } \\
\hline Nominal input (peak input) & $+/-1.25 \mathrm{mT}(+/-3.75 \mathrm{mT})$ \\
\hline Nom. Output (Peak output) & $+/-2 \mathrm{~mA}(+/-6 \mathrm{~mA})$ \\
\hline Sensitivity & $1.6 \mathrm{~mA} / \mathrm{mT}$ \\
\hline $\begin{array}{l}\text { Bandwidth / } \\
\text { step response time }\end{array}$ & $\begin{array}{l}500 \mathrm{kHz} / \\
<1 \mu \mathrm{s}\end{array}$ \\
\hline Output noise (@ BW) & $1.5 \mu \mathrm{A}_{\mathrm{rms}}(\mathrm{BW}=1 \mathrm{~Hz} \ldots 20 \mathrm{kHz})$ \\
\hline Sig.Nom / Nrms & 1333 \\
\hline Offset in Temp (\% Nominal) & $+/-0.75 \%$ \\
\hline Sensitivity drift (in Temp) & $<+/-1.0 \%$ \\
\hline Linearity error (\% Nominal) & $<+/-0.3 \% @$ OUTn \\
\hline \hline
\end{tabular}

* The linearity error refers to the nominal output of $2 \mathrm{~mA}$, also for the peak output of $6 \mathrm{~mA}$.

Table 2: target performance key parameters for IACS 
The SNR of $63 \mathrm{db}$ is the base of the parameter sig. Nom/Nrms $=1333$. With this, a magnetic field gradient of 1.2 $\mathrm{A} / \mathrm{m} / \mathrm{mm}$ can be resolved, if the target value of $1600 \mathrm{~A} / \mathrm{m} / \mathrm{mm}$ is reached at $I_{\text {nom. }}$ A current in the range of $100 \mathrm{~mA}$ could be resolved for the above example in Fig. 15.

\section{First Results}

The following figures show first measurements of engineering samples with all system components enclosed within one SOIC16 package. In Fig. 16 the high dynamic of the system is demonstrated.

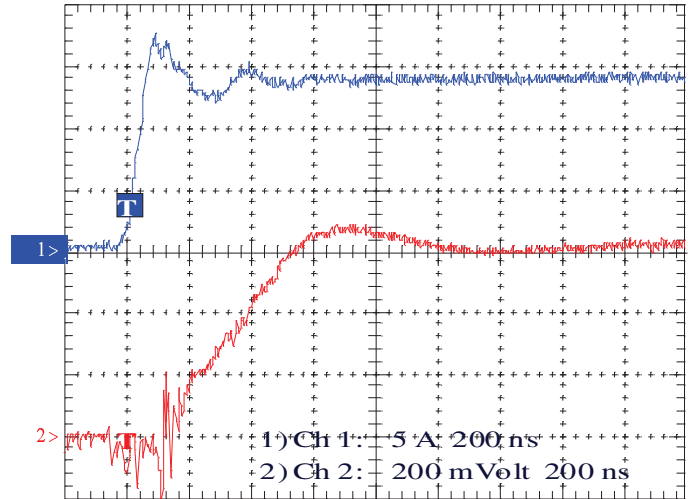

Fig. 16: signal response. Rise Time $<600$ ns

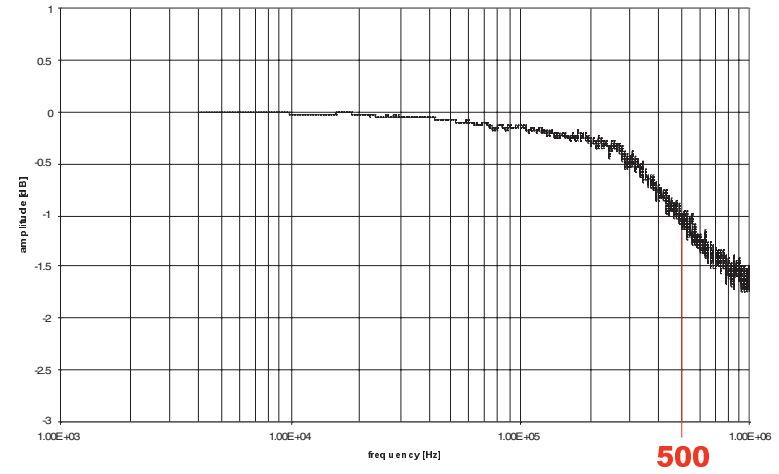

Fig. 17: IACS bandwidth: $-3 \mathrm{~dB}$ is above $500 \mathrm{kHz}$

First measurements of the bandwidth in Fig. 17 show that the targeted bandwidth of $500 \mathrm{kHz}$ is exceeded.

\section{Pre-qualification}

The system was pre-qualified under different environmental conditiones. This includes high temperature storage $\left(150^{\circ} \mathrm{C}\right.$, temperature-humidity-biased test at $85 \%{ }^{\circ} \mathrm{C}$ and $85 \%$ rel. humidity, as well as 1000 temperature shocks from $-50^{\circ} \mathrm{C}$ to $150^{\circ} \mathrm{C}$. In total, 145 samples were tested, and all were pass after the pre-qualification.

\section{Application Example}

Fig. 18 show the use of the IACS for a three phases rectifier for high currents ( 200 A).
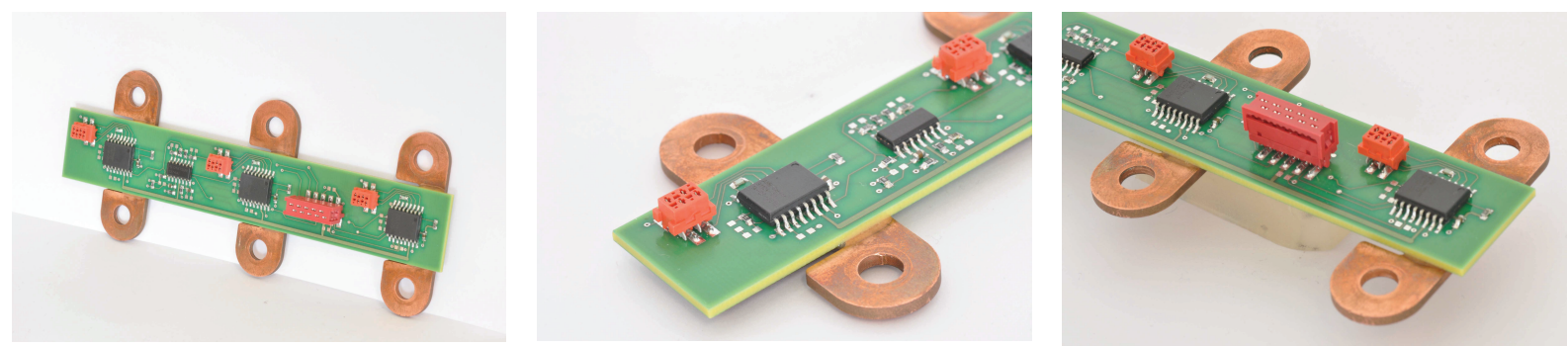

Fig. 18: three phase rectifier for high currents

This example shows the benefit of the external current bar concept: the PCB is fabricated with standard methods, with also the current sensors attached by standard reflow soldering. The primary current bar is fabricated independently from the PCB.

\section{Conclusion}

A new current sensor system, based on the AMR-effect, has been developed. Due to its external primary current bar concept, it can be adapted to a wide range of applications. Sensor chip, readout circuitry and biasing magnets are mounted inside a standard JEDEC SOIC16 package. First measurements show its targeted high dynamic range. The feasibility of this packaging concept was prooven by a pre-qualification. 